\title{
Prevalência de ideação suicida em acadêmicos da área de saúde
}

\author{
Prevalence of suicide ideation in healthcare academics
}

Prevalencia de la ideación del suicidio en académicos del área de salud

\begin{abstract}
Nadja Maria dos Santos ${ }^{1 *}$, Marcus Vinícius dos Santos Faustino1, Maiana da Silva Santana ${ }^{1}$, Flávia Emília Cavalcante Valença Fernandes ${ }^{1}$, Luciana Pessoa Maciel Diniz ${ }^{1}$, Roberto Luís Pereira dos Santos².
\end{abstract}

\section{RESUMO}

Objetivo: Estimar a prevalência de ideação suicida em acadêmicos da área de saúde. Métodos: Trata-se de um estudo descritivo de caráter quantitativo de corte transversal, realizado através da coleta de dados a partir de um questionário semiestruturado e do Inventário Depressão de Beck (IDB). Participaram da pesquisa principal 224 acadêmicos, deste foram selecionados 80 questionários, a análise foi feita no programa estatístico Stata versão 14.0 em que foi realizado o cálculo de média, desvio padrão e cálculo da frequência média absoluta e relativa. Resultados: Dos 80 acadêmicos que apresentaram ideação suicida, a prevalência foi de $36 \%$, verificou-se que a média de idade foi de 20 anos. Os fatores associados a ideação suicida foram: sexo, depressão moderada a grave e histórico de comportamento suicida. Conclusão: Há a prevalência de ideação suicida em acadêmicos da área de saúde evidenciada pelos fatores associados acrescidos da depressão grave. Esses achados demonstram a necessidade da criação de espaços na universidade que promovam a escuta, o acolhimento, como também medidas que visem a preservação da saúde mental dos acadêmicos.

Palavras-chave: Estudantes de ciências da saúde, Ideação suicida, Tentativa de suicídio.

\begin{abstract}
Objective: To estimate the prevalence of suicidal ideation in health academics. Methods: This is a descriptive quantitative cross-sectional study, carried out by collecting data from a semi-structured questionnaire and the Beck Depression Inventory (IDB). 224 academics participated in the main research, 80 questionnaires were selected from this, the analysis was made using the statistical program Stata version 14.0 in which the calculation of mean, standard deviation and calculation of the absolute and relative average frequency was carried out. Results: Of the 80 students who had suicidal ideation, the prevalence was $36 \%$, it was found that the average age was 20 years. The factors associated with suicidal ideation were: sex, moderate to severe depression and a history of suicidal behavior. Conclusion: There is a prevalence of suicidal ideation in health academics evidenced by the associated factors plus severe depression. These findings demonstrate the need to create spaces at the university that promote listening, welcoming, as well as measures aimed at preserving the mental health of academics.
\end{abstract}

Keywords: Health science students, Suicidal ideation, Suicide attempt.

\section{RESUMEN}

Objetivo: Estimar la prevalencia de ideación suicida en académicos de la salud. Métodos: Se trata de un estudio descriptivo, cuantitativo, transversal, realizado mediante la recolección de datos de un cuestionario semiestructurado y del Inventario de Depresión de Beck (BID). 224 académicos participaron en la investigación principal, de esta se seleccionaron 80 cuestionarios, el análisis se realizó mediante el programa estadístico Stata versión 14.0 en el cual se calculó la media, desviación estándar y frecuencia promedio absoluta y relativa. Resultados: De los 80 estudiantes que tuvieron ideación suicida, la prevalencia fue del

1 Universidade Estadual de Pernambuco (UPE), Petrolina - PE. *E-mail: nadja.santos@upe.br

2 Instituto de Medicina Holística (HOLOS), Petrolina - PE. 
$36 \%$, se encontró que la edad promedio fue de 20 años. Los factores asociados a la ideación suicida fueron: sexo, depresión moderada a severa y antecedentes de conducta suicida. Conclusión: Existe una prevalencia de ideación suicida en los académicos de la salud evidenciada por los factores asociados más la depresión severa. Estos hallazgos demuestran la necesidad de crear espacios en la universidad que promuevan la escucha, la acogida, así como medidas que tengan como objetivo preservar la salud mental de los académicos.

Palabras clave: Estudiantes de ciencias de la salud, Ideación suicida, Intento de suicidio.

\section{INTRODUÇÃO}

O suicídio apresenta-se como uma das vinte principais causas de morte no mundo, superando o número de óbitos por razões como guerra e homicídio, e alcançando aproximadamente 800.000 atos consumados a cada ano, sendo a segunda causa de morte entre indivíduos na faixa etária de 15 a 29 anos no mundo (OMS, 2019). No Brasil, observa-se uma maior prevalência de casos notificados de lesão autoprovocada, assim como as tentativas e suicídio, essas se encontram na faixa etária entre os 20 e 49 anos; em 2018, foram registrados 12.773 óbitos por suicídio e 9.438 internações por lesões autoprovocadas (BRASIL, 2018).

O suicídio se configura como algo que está presente na vida e no cotidiano das pessoas, apesar de que, na maioria das vezes opta-se por ignora-lo. Este tem sido apontado como um problema com predições preocupantes, e que tem se tornado uma questão de saúde pública (OMS, 2014).

Segundo a Organização Mundial da Saúde o comportamento suicida compreende a ideação, o planejamento, a tentativa e o suicídio consumado. A tentativa de suicídio é definida como qualquer comportamento suicida não letal, como o envenenamento, a lesão ou autoagressão com ou sem intenção ou resultado fatal. Já o suicídio corresponde ao ato de se matar deliberadamente (OMS, 2014).

Os principais fatores de risco ao suicídio são os eventos estressores que ocorrem ao longo da vida, como trauma ou abuso, a falta de apoio social, as perdas, questões de orientação sexual, problemas familiares, estresse social, desesperança, dor, histórico familiar de suicídio e transtornos mentais. Alguns transtornos psíquicos estão mais associados ao suicídio, dentre eles a depressão e o transtorno do humor bipolar. (OMS, 2014; BRAGA LL e DELL'AGLIO DD 2013; SENA F, et al. 2014; VASCONCELOS-RAPOSO J, et al. 2016).

A Grande maioria dos suicídios não ocorrem sem aviso. O tentante na maioria das vezes, demonstra a intenção de matar-se, observa-se também a automutilação, comportamento esse que deve servir de alerta para familiares e profissionais de saúde. Os sentimentos conflitantes é outra característica do tentante que, na maioria das vezes não deseja matar-se, mas quer evidenciar um sofrimento e os problemas enfrentados. Com isso, é necessário monitoramento redobrado aos tentadores, pois quando estão com as energias renovadas, tendem a transformar pensamentos em ação destrutiva (WHO, 2014).

No início da vida universitária surge a mudança brusca de ambiente, muitas vezes, não há o apoio dos pais. Os acadêmicos tornam-se repentinamente responsáveis por todos os atos, tendo que aprender a se virar sozinhos e muitas vezes enfrentam dificuldades sem nenhum auxílio. Por estarem mais vulneráveis, os acadêmicos, podem ser acometidos por patologias como depressão e ansiedade, o que pode vir a acarretar o comportamento suicida, gerado pela pressão imposta pela sociedade, como também pela incapacidade de reagir a essa pressão de forma positiva (CREMASCO GS e BAPTISTA MN, 2017).

Os fatores protetores do suicídio estão associados ao apoio familiar e à confiança; são exemplos desses fatores: as boas habilidades sociais, a procura por ajuda e aconselhamento, o senso de valor pessoal, a abertura para novas experiências. A universidade deve estar atenta a vulnerabilidade emocional do estudante universitário, essa por sua vez não deve ser ignorada. As instituições de ensino superior devem construir dispositivos e implementar iniciativas que beneficiem a integração do estudante com o novo ambiente. Quando o estudante se depara com uma rede de apoio e um ambiente acolhedor favorece a prevenção do suicídio (VAZ RA e VAZ WM, 2019).

Os jovens atualmente, cada vez mais se afastam dos fatores protetores e consequentemente se aproximam dos fatores de risco. Os estudantes universitários, particularmente, tendem a se aproximar desses 
fatores com uma velocidade maior, devido a frequência em lidar com motivos que anulam sua autoestima, a diminuição das redes de apoio, o que aumenta o nível de apreensão, tornando mais vulnerável a desenvolver transtornos mentais e ao consumo de drogas e, isso vai fazer com que esse estudante em detrimento das razões associadas ao ambiente acadêmico perca a esperança e motivação (MOTA RS, et al., 2019).

Vaz RA e Vaz WM (2019), no seu estudo traz que a obrigação de fazer escolhas, a inquietação com o futuro, sentimentos de inadequação, além do tempo exíguo para a execução das demandas, tornam o ambiente universitário propício a tensões de diversas ordens, deixando o indivíduo susceptível e predisposto ao adoecimento psíquico. A vivência advinda desses fatores estressantes sem a devida problematização e a falta de serviços assistenciais para lidar com a angústia podem naturalizar o sofrimento no ambiente universitário, causando uma sensação de desamparo.

Ademais, diante das inúmeras modificações que se caracterizam a contemporaneidade, a academia passou a representar um espaço de possível sofrimento psíquico e provável adoecimento (DINIZ MFPS e AIRES S, 2018). Os transtornos mentais são comuns em universitários e, estão relacionados ao desgaste (CUIJPERS P, et al., 2015). Alguns fatores podem deixar os acadêmicos predispostos ao adoecimento mental, como as cobranças exercidas por si próprio, docentes, familiares e a realização de exercícios avaliativos (SANTOS LA e KIND L, 2020).

Perante o exposto, considerando que a universidade é um ambiente que gera mudanças, observa-se o aumento das responsabilidades, as cobranças e o estresse. E que essas, por sua vez, podem vir a provocar nos acadêmicos um sentimento de incapacidade, de grande tristeza e adoecimento mental justifica-se assim a realização do estudo (OLIVEIRA MF e ARAUJO LMB, 2019).

Além do mais, a pesquisa poderá propiciar um maior conhecimento sobre essa questão de saúde pública global, bem como gerar indicadores sobre a saúde mental dos universitários. O conhecimento desses indicadores contribuirá na elaboração de estratégias de prevenção e acompanhamento, como também medidas que possam minimizar e/ou evitar que esses estudantes desenvolvam transtornos psicológicos que possam levar a decisão de realizar uma atitude irreversível que é o suicídio. Nessa perspectiva, o objetivo deste estudo foi estimar a prevalência de ideação suicida em acadêmicos da área de saúde.

\section{MÉTODOS}

Trata-se de um estudo descritivo de caráter quantitativo de corte transversal. A presente pesquisa integra o projeto: "Ansiedade e depressão em acadêmicos de saúde e fatores associados" realizado em uma Instituição Pública de Ensino Superior (IES), localizada no município de Petrolina, interior do estado de Pernambuco no período de outubro a fevereiro de 2020.

Dos 569 estudantes dos cursos de saúde, participaram da pesquisa principal 224 acadêmicos, os critérios de inclusão foram os questionários e os IDB dos acadêmicos que marcaram sim para ideação suicida, da amostra total foram selecionados 80 questionários.

Os dados foram compilados e codificados no programa Excel $\AA^{1} 16.0$ em duas etapas: a primeira a partir dos dados do IDB e, a segunda, a partir dos dados do questionário semiestruturado, em seguida foram transportados para análise no programa estatístico Stata versão 14.0. Para análise descritiva das variáveis quantitativas foram utilizados o cálculo de média, desvio padrão e cálculo da frequência absoluta e relativa.

Para calcular a significância estatística das associações foi utilizado a regressão logística binária multivariada. A seleção das variáveis no modelo se deu por meio de análise bivariada em que se incluíram as variáveis cujo valor de $p<0,20$. Consideraram-se associados à intenção de tirar a própria vida os fatores cujos valores de $p<0,05$ e seus efeitos apresentados pela razão de chance (Odds Ratio).

A pesquisa foi aprovada pelo Comitê de Ética em Pesquisa do CISAM - Centro Integrado de Saúde Amaury de Medeiros/Universidade de Pernambuco - UPE, sob o parecer no 3.532 .828 e obedeceu aos aspectos éticos determinados na Resolução no 466 de 2012, do Conselho Nacional de Saúde. (BRASIL, 2012). 


\section{RESULTADOS}

A prevalência de ideação suicida entre os acadêmicos foi de $36 \%$. Os achados mostram o perfil dos acadêmicos, a média de idade dos acadêmicos de 20 anos (mínimo 17, máximo 33; desvio padrão 2,4; IC $95 \% 20,23-21,37)$. Dos participantes do estudo $35(43,8 \%)$ cursavam enfermagem, $23(28,8 \%)$ fisioterapia e $22(27,4 \%)$ nutrição respectivamente. Observou-se a predominância do sexo feminino 66 (93\%), e estado civil solteiro 76 (95\%). Com relação a renda familiar destacou a menor que dois salários mínimos 53 (68,8\%). Quanto à religião, constatou-se que $68(87,2 \%)$ tinha religião. No tocante a orientação sexual, $65(84,45 \%)$ informou ser heterossexual.

Tais dados, permitiram fazer a caracterização dos acadêmicos com pensamentos suicidas de uma Instituição Pública de Ensino Superior (Tabela 1).

Tabela 1 - Caracterização dos acadêmicos com pensamentos suicidas de uma Instituição Pública de Ensino Superior.

\begin{tabular}{lcccc}
\hline Variáveis & N & $\%$ & \multicolumn{2}{c}{ IC 95\% } \\
\hline Curso & & & 33,1 & 55 \\
\hline Enfermagem & 35 & 43,8 & 19,7 & 39,8 \\
Fisioterapia & 23 & 28,8 & 18,7 & 38,5 \\
Nutrição & 22 & 27,4 & & \\
\hline Sexo & & & & 93,9 \\
\hline Feminino & 66 & 93 & 2,9 & 16,1 \\
Masculino & 5 & 7 & & \\
\hline Estado civil & & & & 1,8 \\
\hline Outro & 4 & 5 & 57,2 & 98,2 \\
Solteiro & 76 & 95 & 21,7 & 42,6 \\
\hline Renda familiar & & & & \\
\hline Menor que 2 salários mínimos & 53 & 68,8 & 6,9 & 22,5 \\
Maior ou igual a 2 salários mínimos & 24 & 31,2 & 7,5 & 93,1 \\
\hline Tem alguma religião & & & & \\
\hline Não & 10 & 12,8 & 74,3 & 91 \\
Sim & 68 & 87,2 & 3,5 & 16,5 \\
\hline Orientação sexual & & & 1,2 & 11,7 \\
\hline Heterossexual & 65 & 84,4 & 9,2 & 9 \\
Homossexual & 6 & 7,8 & 0,2 & 9 \\
Bissexual & 3 & 3,9 & 0,2 & 9 \\
Trans LGBTQ+ & 1 & 1,3 & & \\
Indefinido & 1 & 1,3 & 1,3 & \\
Pansexual & 1 & 100 & & \\
\hline Total & 80 & & & \\
\hline Fonte: Santos NM, et al, 2020., & & & \\
\hline
\end{tabular}

Fonte: Santos NM, et al., 2020.

No que concerne aos dados do IDB dos acadêmicos que marcaram sim para o comportamento suicida, conforme descrito abaixo, na Tabela 2, 35 (43,8\%) apresentaram depressão leve a moderada, 22 (27,5\%) depressão moderada a grave e $8(10,0 \%)$ depressão grave respectivamente, essa variável é considerada um fator de risco, revelando que $65(81,30 \%)$ da população do estudo apresentavam sinais da doença. Quanto a distribuição dos dados psicológicos/psiquiátricos, $72(90 \%)$ informou não fazer tratamento psicológico. Com relação ao histórico familiar de doença mental, a grande maioria $51(64,6 \%)$ respondeu não. No que diz respeito a história pregressa de comportamento suicida pelo acadêmico, 61 (78,2\%) respondeu não. Dos acadêmicos que informaram histórico de comportamento suicida os meios de perpetração utilizados foram: não especificados 5 (23,8\%), autointoxicação $4(19,1 \%)$ e dois ou mais meios $4(19,1 \%)$. 
Quando questionados se já tinham pensado em dormir e nunca mais acordar 48 (60\%) informou que sim, com relação ao tempo disseram que tiveram essa vontade há mais de 3 meses. O motivo assinalado pelos acadêmicos no que diz respeito a vontade de dormir e nunca mais acordar foi o de acabar com o sofrimento, dado esse que representou $51(91,1 \%)$ dos respondentes (Tabela 2).

Tabela 2 - Dados psicológicos/psiquiátricos dos acadêmicos de uma Instituição Pública de Ensino Superior com pensamentos suicida.

\begin{tabular}{|c|c|c|c|c|}
\hline \multirow{2}{*}{$\begin{array}{l}\text { Variáveis } \\
\text { IDB }\end{array}$} & \multirow[t]{2}{*}{$\mathbf{n}$} & \multirow[t]{2}{*}{$\%$} & \multicolumn{2}{|c|}{ IC 95\% } \\
\hline & & & & \\
\hline Ausência de depressão ou depressão mínima & 15 & 18,8 & 11,5 & 29,1 \\
\hline Depressão leve a moderada & 35 & 43,8 & 33,1 & 55 \\
\hline Depressão moderada a grave & 22 & 27,4 & 18,7 & 38,5 \\
\hline Depressão grave & 8 & 10 & 5 & 19 \\
\hline \multicolumn{5}{|l|}{ Faz tratamento com psicólogo } \\
\hline Não & 72 & 90 & 81 & 95 \\
\hline Sim & 8 & 10 & 5 & 19 \\
\hline \multicolumn{5}{|l|}{ Já fez uso de algum fármaco psicoativo } \\
\hline Não & 64 & 80 & 69,6 & 87,5 \\
\hline Sim & 16 & 20 & 12,5 & 30,4 \\
\hline \multicolumn{5}{|l|}{ Histórico de doença mental na família } \\
\hline Não & 51 & 64,6 & 53,2 & 74,5 \\
\hline Sim & 28 & 35,4 & 25,5 & 46,8 \\
\hline \multicolumn{5}{|l|}{ Histórico de comportamento suicida } \\
\hline Não & 61 & 78,1 & 67,4 & 86,1 \\
\hline $1 \mathrm{vez}$ & 8 & 10,3 & 5,1 & 19,4 \\
\hline 2 vezes & 3 & 3,9 & 1,2 & 11,5 \\
\hline 3 vezes & 4 & 5,1 & 1,9 & 13,1 \\
\hline 5 vezes ou mais & 2 & 2,6 & 0,6 & 10 \\
\hline \multicolumn{5}{|l|}{ Qual meio de perpetração } \\
\hline Autointoxicação & 4 & 19,1 & 6,7 & 43,6 \\
\hline Enforcamento e sufocação & 2 & 9,5 & 2,1 & 34 \\
\hline Objeto cortante ou penetrante & 3 & 14,3 & 4,2 & 38,7 \\
\hline Precipitação de um lugar elevado & 2 & 9,5 & 2,1 & 34 \\
\hline Precipitação de um meio em movimento & 1 & 4,7 & 0,6 & 30,9 \\
\hline Meios não especificados & 5 & 23,8 & 9,5 & 48,3 \\
\hline 2 ou mais meios de perpetração & 4 & 19,1 & 6,7 & 43,6 \\
\hline \multicolumn{5}{|l|}{ Já pensou em dormir e nunca mais acordar } \\
\hline Sim (nos últimos 3 meses) & 32 & 40 & 29,7 & 51,3 \\
\hline Sim (a mais de 3 meses) & 48 & 60 & 48,7 & 70,3 \\
\hline \multicolumn{5}{|l|}{ Qual motivo } \\
\hline Para chamar atenção & 4 & 7,1 & 2,6 & 18 \\
\hline Para acabar com o sofrimento & 51 & 91,1 & 79,8 & 96,3 \\
\hline Ambas as opções & 1 & 1,8 & 0,2 & 12,3 \\
\hline Total & 80 & - & & \\
\hline
\end{tabular}

Fonte: Santos NM, et al., 2020.

Observou-se que os fatores que estiveram associados ao comportamento suicida foram: sexo, presença de depressão moderada a grave, depressão grave e histórico de comportamento suicida. Dentre o fator sexo, o masculino apresentou uma menor chance de desenvolver um novo episódio de ideação suicida $(O R=0,11$; p-valor: 0,014) (Tabela 3). 
Para os acadêmicos com depressão, os que apresentaram depressão moderada a grave, maior foi a chance de ter nova ideia suicida $(O R=5,61 ; p$-valor: 0,005$)$ assim como a presença depressão grave (OR= 11,93; p-valor: 0,045). Os acadêmicos com histórico de comportamento suicida também apresentaram mais chance de ter nova ideia suicida $(O R=27,59$; $p$-valor: 0,002) (Tabela 3).

Tabela 3 - Análise multivariada associada a ideação suicida em acadêmicos de uma Instituição Pública de Ensino Superior.

\begin{tabular}{|c|c|c|c|c|}
\hline \multirow{2}{*}{$\begin{array}{l}\text { Variáveis } \\
\text { Sexo }\end{array}$} & \multirow[t]{2}{*}{ Odds Ratio } & \multirow[t]{2}{*}{ p-valor } & \multicolumn{2}{|c|}{ IC95\% } \\
\hline & & & & \\
\hline Masculino & 0,11 & 0,014 & 0,02 & 0,64 \\
\hline Feminino & 1 & & & \\
\hline Média de idade & 0,88 & 0,112 & 0,76 & 1,03 \\
\hline \multicolumn{5}{|l|}{ Percepção de Bem estar } \\
\hline Péssimo/ruim/regular & 0,8 & 0,711 & 0,25 & 2,59 \\
\hline Bom/ ótimo & 1 & & & \\
\hline \multicolumn{5}{|l|}{ Percepção da saúde } \\
\hline Péssimo/ruim/regular & 1,07 & 0,91 & 0,35 & 3,25 \\
\hline Bom/ ótimo & 1 & & & \\
\hline \multicolumn{5}{|l|}{ Percepção do sono } \\
\hline Ruim & 1,66 & 0,291 & 0,65 & 4,23 \\
\hline Bom/ ótimo & 1 & & & \\
\hline \multicolumn{5}{|l|}{ Depressão (IDB) } \\
\hline Ausência de depressão & 1 & & & \\
\hline Depressão leve a moderada & 1,12 & 0,806 & 0,45 & 2,81 \\
\hline Depressão moderada a grave & 5,61 & 0,005 & 1,69 & 18,68 \\
\hline Depressão grave & 11,93 & 0,045 & 1,05 & 134,99 \\
\hline \multicolumn{5}{|l|}{ Cobrança dos docentes } \\
\hline Alto/muito alto/extremamente alto & 1,03 & 0,969 & 0,28 & 3,7 \\
\hline Baixo/médio & 1 & & & \\
\hline \multicolumn{5}{|l|}{ Assistir televisão } \\
\hline Sim & 0,82 & 0,738 & 0,25 & 2,7 \\
\hline Não & 1 & & & \\
\hline \multicolumn{5}{|l|}{ Vai ao cinema } \\
\hline Sim & 0,64 & 0,468 & 0,19 & 2,15 \\
\hline Não & 1 & & & \\
\hline \multicolumn{5}{|l|}{ Usa computador } \\
\hline Sim & 0,22 & 0,259 & 0,02 & 3,04 \\
\hline Não & 1 & & & \\
\hline \multicolumn{5}{|l|}{ História familiar de doença mental } \\
\hline Sim & 1,03 & 0,951 & 0,43 & 2,44 \\
\hline Não & 1 & & & \\
\hline \multicolumn{5}{|l|}{ Uso de maconha } \\
\hline Sim & 11,45 & 0,096 & 0,65 & 201,8 \\
\hline Não & 1 & & & \\
\hline \multicolumn{5}{|l|}{ Cobrança pessoal } \\
\hline Alto/muito alto/extremamente alto & 1,12 & 0,836 & 0,4 & 3,15 \\
\hline Baixo/médio & 1 & & & \\
\hline Histórico suicídio & 27,59 & 0,002 & 3,47 & 219,65 \\
\hline
\end{tabular}

Fonte: Santos NM, et al., 2020. 


\section{DISCUSSÃO}

No presente estudo foi evidenciado a prevalência de ideação suicida nos acadêmicos, onde $36 \%$ idealizam o suicídio nos últimos três meses. Prevalências inferiores foram encontradas em estudos similares, com acadêmicos de saúde em universidade pública em Teresina, PI ( $n=142)$ onde 22\% dos estudantes apresentaram ideação suicida Veloso LUP, et al., (2019) e o mesmo ocorreu com 9,9\% acadêmicos do Mato Grosso (SANTOS HGB, et al., 2017).

Os fatores associados à ideação suicida com o público universitário, pesquisado neste estudo, corroboram com os achados na população em geral (WHO, 2014). Um estudo de revisão realizado com 31 artigos, publicados entre 1959 e 2001, identificou que em 97\% dos 15.629 casos de suicídio ocorridos no hemisfério norte, caberia algum diagnóstico (BOTEGA N, 2015). No Brasil, os fatores de risco para o suicídio são transtorno mental, abuso de álcool e outras drogas, relação familiar conturbada e tentativa de suicídio prévia (GOMES E, et al., 2019). Esses dados corroboram parcialmente com os achados desse estudo, onde os fatores de risco encontrados foram a presença de depressão e histórico de comportamento suicida prévio.

Alguns artigos versam o transtorno mental como fator de risco, destacando a depressão como a mais frequente e o suicídio como um agravo decorrente do transtorno (CRUZ CA, et al., 2020). Esse estudo revelou que a depressão foi um fator de risco para a ideação suicida, assim como o encontrado naquele estudo (ASSUNÇÃO WC e SILVA JBF, 2019). A ideação suicida em algumas pessoas portadoras de depressão aparece como um aviso, uma forma de chamar a atenção para o sofrimento enfrentado no momento, buscando uma mudança nas situações vivenciadas (SOUZA C e MOREIRA V, 2018).

Este estudo traz uma prevalência maior de comportamento suicida no sexo feminino, entre solteiros e com renda familiar abaixo de 2 salários mínimos. Em um estudo realizado no Brasil sobre comportamento suicida demonstrou também maior incidência do suicídio em mulheres (GOMES CFM e SILVA DA, 2020). Em Portugal, em um estudo realizado para comparar os níveis de ideação suicida entre estudantes universitários, observou-se que não houve diferença entre solteiros e casados e com relação a renda a maioria recebiam menos que dois salários mínimos, a informação encontrada neste estudo condiz com os achados dessa pesquisa (VASCONCELOS-RAPOSO J, et al., 2016). O estudo de Feltrin RJ e Almeida FJH (2020), mostrou que a taxa de suicídio diminui quando a renda se eleva e o de Moreira BC e Melo MMA (2019) trouxe que a incidência era maior em pessoas com renda familiar maior que 5 salários mínimos.

Em um estudo realizado com estudantes universitários do estado do Mato Grosso para identificar os fatores associados a ideação suicida, trouxe que a prática religiosa atuava como um fator de proteção para a ideação, nesse estudo, não foi avaliado a relação da ideação suicida e a prática religiosa (SANTOS HGB, et al., 2017).

Richard TL, et al. (2020) no seu estudo descreve que, a prevalência de comportamento suicida nos jovens LGBTQ+ persiste durante décadas, observando-se uma queda no período de 1995 a 2017, porém ainda permanece alta quando comparada com as pessoas heterossexuais, os achados desse estudo mostraram que os jovens LGBTQ+ foram a minoria entre os acadêmicos.

Em relação ao tratamento psicológico, os dados mostram que $90 \%$ dos acadêmicos com ideação suicida não faziam tratamento psicológico, essa situação pode ser altamente danosa para alguém que está em sofrimento psíquico. Essa afirmação corrobora com Reis AH, et al. (2020), que diz ser necessária a ajuda psicológica, porém ainda existe um grande tabu na sociedade fazendo com que as pessoas evitem buscar tratamento. Sendo que é necessário pensar na integralidade do cuidado que será efetivada pela intersetorialidade levando informação e tratamento adequado para pessoas que necessitem dessa ajuda (SANTOS LA e KIND L, 2020).

E que 20\% desses utiliza algum fármaco psicotrópico, sendo perceptível a automedicação, em um estudo feito em Taubaté entre 2014 e 2018 afirma que as maiores causas de intoxicação foram relacionadas a automedicação principalmente na população entre 20 e 39 anos (MOTA SF, et al., 2020). 
Sobre o histórico familiar de doença mental, neste estudo cerca de $25,4 \%$ dos entrevistados afirmaram ter familiar portador de transtorno, esse dado pode influenciar para o aparecimento de doenças como depressão, ansiedade e a ideação suicida; no estudo realizado sobre a hereditariedade e repetição de ato suicida, Lima FN e Gondim DSM (2019) afirmou que a pessoa adquire traços de pessoas amadas, como pai, mãe e parentes próximos, sendo um dos fatores para o desenvolvimento da intenção suicida.

Foi evidenciado nesta pesquisa que o método utilizado para tirar a própria vida foi a autointoxicação, esse é um dos métodos menos letais, porém mais utilizados pelas mulheres (MIRANDA CCS, et al., 2020). Segundo Silva AR, et al., (2014), as intoxicações representam um grande problema de saúde pública, devido ao aumento considerável de tentativas de suicídio utilizando esse método.

Com relação a vontade de dormir e nunca mais acordar, observou-se que o motivo seria o de acabar com o sofrimento, sentimento esse muitas vezes encoberto nas diversas atividades realizadas pelo ser universitário, que acaba por silenciar essa dor habitável em suas vidas, ficando aprisionada em uma interioridade sem som, possibilitando o anuncio do fim de tudo (SILVA MVM e AZEVEDO AKS, 2018).

Quando avaliada as variáveis relacionadas ao lazer, assistir tv, ir ao cinema, usar o computador e a ideação suicida, não apresentaram significância estatística; contrariando o estudo de Albuquerque CS e Matos RA (2020), onde afirmou que pessoas que praticam poucas vivencias de lazer tem uma predisposição maior para desenvolver pensamentos suicidas. Um outro estudo realizado com universitários na China, Lew B, et al., (2019) trouxe que a idade, em média 19 anos, que esses jovens saem de casa para estudar, a falta de tempo para atividades de lazer e a sobrecarga de trabalhos na academia, foram fatores de risco preditores para o pensamento suicida.

Sentimentos vivenciados no período da universidade, como também os dos períodos anteriores se tornam gatilhos para que os acadêmicos tenham a vontade de dar um fim a sua própria vida, uma vez que esses sentimentos diminui o rendimento acadêmico, consequentemente acarreta um desespero e vazio existencial, levando-o a questionar a existência da própria vida (SILVA MVM e AZEVEDO AKS, 2018).

Observou-se que a cobrança dos docentes relacionada ao comportamento suicida também não foi significante; o estudo de Fernandes MA, et al., (2020), aponta que a cobrança que o acadêmico impõe a si na busca de melhores resultados pode levar ao desenvolvimento de ansiedade.

Pessoas que apresentam problemas para dormir e sono inadequado, apresentam uma maior probabilidade de planejar e consumar o ato suicida (ALVES JCAS, et al., 2016). Nesse estudo, não houve associação da qualidade de sono com a ideação suicida.

As tentativas de suicídio muitas vezes são realizadas para evidenciar o problema pelo qual está passando para um ente querido; essas tentativas são chamadas de falsas tentativas, são métodos irreais facilmente reversíveis, na maioria das vezes são realizadas para que fique evidente o sofrimento psíquico e seu descontentamento com a vida, anunciando assim o seu desejo de morte (FREITAS APA e BORGES LM, 2017). Para a consumação do ato suicida, as tentativas se mostram fatores de risco bastante importante sendo que quanto mais tentativas existirem, mais provável pode se tornar a consumação do ato suicida (ROSA NM, et al., 2016).

A repercussão do suicídio aos familiares e amigos envolve prejuízos emocionais, sociais e econômicos, principalmente quando evidenciado em faixas etárias mais jovens. Diante do exposto, é evidente a necessidade da enfermagem aprimorar suas competências, que vão desde a avaliação até realização de cuidados específicos, no intuito de enfrentar este crescente problema de saúde pública (TRIGUEIRO A, 2015).

O presente estudo teve como limitações a dificuldade de encontrar os acadêmicos na universidade durante a aplicação dos questionários, por estarem em período de prática nos serviços de saúde; balizando assim a possibilidade de estimar e caracterizar melhor a prevalência de ideação suicida e os fatores associados nos acadêmicos. 


\section{CONCLUSÃO}

Os fatores determinantes da ideação suicida em acadêmicos de saúde foram: sexo, presença de depressão moderada a grave, depressão grave e histórico de comportamento suicida, aumentando as chances de ocorrência de novas tentativas suicidas. Observou-se uma maior prevalência de comportamento suicida entre os estudantes de enfermagem, o sexo feminino, o estado civil solteiro e a renda familiar menor que dois salários mínimos. Alguns achados não foram significantes na ocorrência da ideação suicida, tais como: a cobrança exercida pelos docentes e a participação em algumas atividades de lazer. Tais achados reafirmam aos indícios da literatura e demonstram a necessidade de criação de espaços na universidade que promovam a escuta, o acolhimento, como também a implantação de medidas que visem a preservação da saúde mental desses acadêmicos.

\section{REFERÊNCIAS}

1. ALBUQUERQUE CS, MATOS RA. Produção social do suicídio e "questão social" na realidade de Iguatu (CE). Argumentum, 2020; 12 (2): 220-237.

2. ALVES JCAS., et al. Suicidal behaviour in adolescents: Characteristics and prevalence. J Hum Growth, 2016; 26(1): 88-94.

3. ASSUNÇÃO WC, SILVA JBF. Depressão e Suicídio sob a Perspectiva da Psicologia Cognitivo-Comportamental. Revista Saúde em Foco, 2019; 6 (1): 85-102.

4. BARROS PDQ, et al. Associação entre o consumo de drogas e a ideação suicida em adolescentes. Mental, $2017 ; 11$ (21): 304-320.

5. BOTEGA N. Crise suicida: avaliação e manejo. 2015, Porto alegre: Artmed.

6. BRAGA LL, DELL'AGLIO DD. Suicídio na adolescência: fatores de risco, depressão e gênero. Contextos Clínic, 2013; $6(1): 2-14$.

7. BRASIL. Ministério da Saúde. Conselho Nacional de Saúde. Resolução n. 466, de 12 de dezembro de 2012. Aprova diretrizes e normas regulamentadoras de pesquisas envolvendo seres humanos. Brasília, Diário Oficial da União, 12 dez. 2012.

8. BRASIL. Ministério da Saúde: Sistema de Informações Hospitalares (SIH). MORBIDADE HOSPITALAR DO SUS POR CAUSAS EXTERNAS: POR LOCAL DE RESIDÊNCIA. Brasília: Ministério da Saúde, 2018.

9. CREMASCO GP, BAPTISTA MN. Depressão, motivos para viver e o significado do suicídio em graduandos do curso de psicologia. Est. Inter. Psicol, 2017; 8 (1): 22-37.

10. CUIJPERS P, et al. Tratamento psicológico da depressão em estudantes universitários: uma metanálise. Depression Auxiety, 2015; 33 (5): 400-414.

11. CRUZ CA, et al. O Suicídio na Perspectiva das Psicologias Humanista, Fenomenológica e Existencial: Revisão Sistemática e Metassíntese. Contextos Clínicos,2020. 13 (1).

12. DINIZ NFPS, AIRES S. Grupo de escuta e reflexão com estudantes universitários. Revista do NESME, 2018; 15(1): 61-75.

13. FELTRIN RJ, ALMEIDA FJH. Determinantes socioeconômicos do suicídio em Santa Catarina: uma análise com dados em painel, 2018.

14. FERNANDES MA. et al. Prevenção ao suicídio: vivências de estudantes universitários. Revista Cuidarte. 2020; 11(2): e791.

15. FREITAS APA, BORGES LM. Do acolhimento ao encaminhamento: $O$ atendimento às tentativas de suicídio nos contextos hospitalares. Estud. Psicol., 2017; 22 (1): 50-60.

16. GOMES CFM, SILVA DA. Epidemiological aspects of suicidal behavior in college students. Research, Society and Development, 2020; 9(5): e38953106.

17. GOMES E, et al. Revisão integrativa de produções científicas da psicologia sobre comportamento suicida. Revista Psicologia e Saúde,2019; 11(2): 35-53.

18. LEW B, et al. Uma comparação entre estudantes universitários americanos e chineses sobre parâmetros de comportamento relacionados ao suicídio. Jornal Internacional de Psicologia clínica e da Saúde, 2019; 20(2): $108-117$.

19. LIMA FN, GONDIM DSM. Hereditariedade e repetição no ato suicida: uma abordagem psicanalítica. Interdisciplinary Scientific Journal, 2019; 6(5):354.

20. MIRANDA CCS, et al. Perfil epidemiológico dos casos notificados de tentativas de suicídio por intoxicação exógena no estado do Piauí. Research, Society and Development, 2020; 9 (9) e798997862.

21. MOREIRA BC, MELO MMA. Modernidade e suicídio em dois momentos: século XIX e século XXI, 2019; 71ª Reunião Anual da SBPC, UFMS-MS.

22. MOTA SF, et al. Caracterização do perfil das intoxicações medicamentosas na população de Taubaté, São Paulo, no período de 2014 a 2018. Braz. J. Hea. Rev., 2020; 3 (5): 12672-12683.

23. MOTA RS, et al. Aspectos psicossociais desencadeadores de ideação suicidam no ambiente universitário. Projeto de Iniciação Científica concluído - Faculdade de Psicologia, Multivix-Vitória, 2019.

24. OLIVEIRA MF, ARAUJO LMB. Saúde Mental do estudante de medicina. Braz.J.of Develop, $2019 ; 5$ (11):23440-23452. 
25. REIS AH, et al. O suicídio na visão do século XIX e na contemporaneidade - desafios aos paradigmas médico e psicológico. Revista Científica do UniRios, 2020.2; 383-397.

26. RICHARD TL, et al. Ideação suicida e comportamento entre minorias sexuais e jovens heterossexuais: 1995-2017, Pediatrics, 2020; 145 (3): e20192221.

27. ROSA NM, et al. Tentativas de suicídio e suicídios na atenção pré-hospitalar, J.Bras Psiquiatr, $2016 ; 65$ (3): $231-238$.

28. SANTOS LA, KIND L. Integralidade, intersetorialidade e cuidado em saúde: caminhos para se enfrentar o suicídio. Interface (Botucatu), 2020; 24: e190116.

29. SANTOS HGB, et al. Fatores associados à ideação suicida entre estudantes universitários. Rev. LatinoAm Enfermagem [online], 2017; 25: e2878.

30. SILVA AR, et al. Tentativa de suicídio em adolescentes por intoxicação: ações de enfermagem. Trabalho de Conclusão do Curso de Especialização em Linhas de Cuidado em Enfermagem, 2014.

31. SILVA MVM, AZEVEDO AKS. Um olhar sobre o Suicídio: vivências e experiências de estudantes universitários. Revista Psicologia, Diversidade e Saúde, 2018; 7(3): 390-401.

32. SOUZA C, MOREIRA V. Tristeza, depressão e suicídio melancólico: a relação com o Outro. Arquivos Brasileiros de Psicologia, 2018; 70 (2): 173-185.

33. VASCONCELOS-RAPOSO J, et al. Níveis de ideação suicida em jovens adultos. Estudos de Psicologia [Internet]. 2016; 33 (2): 345-354.

34. VELOSO LUP et al. Ideação suicida em universitários da área da saúde: prevalência e fatores associados. Rev. Gaúcha Enferm., 2019; vol.40: e20180144.

35. TRIGUEIRO A. Viver é a melhor opção- A prevenção do suicídio no Brasil e no mundo. 2th. ed. Fraterno: São Bernardo do Campo; 2015.

36. VAZ RA, VAZ WM. O processo de individuação dos estudantes universitários como manejo do comportamento suicida. Pesquisas e Práticas Psicossociais, 2019; 14(4): e3404.

37. WHO. World Health Organization. Preventing suicide - a global imperative. [Internet], 2014. 